MITSUBISHI ELECTRIC RESEARCH LABORATORIES

https://www.merl.com

\title{
An Unsupervised Indoor Localization Method based on Received Signal Strength (RSS) Measurements
}

\author{
Pajovic, M.; Orlik, P.V.; Koike-Akino, T.; Kim, K.J.; Aikawa, H.; Hori, T.
}

TR2015-129 December 06, 2015

\begin{abstract}
We propose an unsupervised, received signal strength (RSS)-based indoor localization method, which as an infrastructure uses commercial WiFi chipsets and does not require any changes in the existing hardware. The method relies on path loss model for measured RSS levels where path loss coefficient is treated as a discrete random variable which takes values from some finite alphabet. The unknown location and path loss coefficient corresponding to each access point are jointly estimated using the Expectation Maximization (EM) approach. The algorithm is experimentally tested in an office space area of dimensions $32-$ by- $52 \mathrm{~m}$ (1600 $\mathrm{m} 2$ ) with only five access points and the achieved average localization error is below $4.5 \mathrm{~m}$.
\end{abstract}

2015 IEEE Global Communications Conference (GLOBECOM) 2015

(C) 2015 MERL. This work may not be copied or reproduced in whole or in part for any commercial purpose. Permission to copy in whole or in part without payment of fee is granted for nonprofit educational and research purposes provided that all such whole or partial copies include the following: a notice that such copying is by permission of Mitsubishi Electric Research Laboratories, Inc.; an acknowledgment of the authors and individual contributions to the work; and all applicable portions of the copyright notice. Copying, reproduction, or republishing for any other purpose shall require a license with payment of fee to Mitsubishi Electric Research Laboratories, Inc. All rights reserved. 



\title{
An Unsupervised Indoor Localization Method based on Received Signal Strength (RSS) Measurements
}

\author{
Milutin Pajovic ${ }^{1}$, Philip Orlik ${ }^{1}$, Toshiaki Koike-Akino ${ }^{1}$, Kyeong Jin Kim ${ }^{1}$, Hideto Aikawa ${ }^{2}$ and Toshinori Hori ${ }^{2}$ \\ ${ }^{1}$ Mitsubishi Electric Research Laboratories (MERL), Cambridge, MA, 02139, USA \\ ${ }^{2}$ Information Technology R\&D Center, Mitsubishi Electric Corporation, 5-1-1 Ofuna, Kamakura, Japan \\ Email: pajovic@merl.com
}

\begin{abstract}
We propose an unsupervised, received signal strength (RSS)-based indoor localization method, which as an infrastructure uses commercial WiFi chipsets and does not require any changes in the existing hardware. The method relies on path loss model for measured RSS levels where path loss coefficient is treated as a discrete random variable which takes values from some finite alphabet. The unknown location and path loss coefficient corresponding to each access point are jointly estimated using the Expectation Maximization (EM) approach. The algorithm is experimentally tested in an office space area of dimensions $32-b y-52 \mathrm{~m}\left(1600 \mathrm{~m}^{2}\right)$ with only five access points and the achieved average localization error is below $4.5 \mathrm{~m}$.
\end{abstract}

\section{INTRODUCTION}

The problem of locating objects and devices within an indoor (enclosed) area has driven much of research attention over the last two decades. Some of many applications include locating people, objects and resources in multilevel garages, hospitals, warehouses, shopping malls, airports, large bus and train stations. The Global Positioning System (GPS), widely used for outdoor localization, is not well suited for indoor localization because GPS signals get attenuated when they propagate thorough indoor environment. At best, the GPS has to be used in conjunction with some other indoor localization technique.

A variety of localization methods have been proposed in the literature. Different physics such as radio waves, sound, motion dynamics, optics, magnetics or their combination, in conjunction with signal processing and machine learning techniques have been explored to map measurements of physical variables into location estimates. Excellent surveys of different indoor localization methods are given in [1] and [2].

A possible classification of indoor localization methods is based on whether or not the development and installation of dedicated hardware is required [3]. While majority of the proposed methods rely to some extent on the deployment of dedicated infrastructure, infrastructure-free methods are viewed as advantageous from a cost perspective. Given the widespread deployment of commercial chipsets from IEEE 802.11 family, both in modern mobile (smart) devices and access points providing internet coverage in indoor areas, $\mathrm{WiFi}$ radio is one of the most prevailing technologies behind the existing infrastructure-free localization methods. In addition, since the measurements of the received signal strength (RSS) levels are easily available in modern WiFi chipsets, indoor localization based on RSS measurements of the WiFi signal has become an attractive approach.

Traditionally, WiFi RSS based indoor localization methods require fingerprinting which essentially creates a training data set consisting of RSS levels measured at known locations in an area of interest. The localization problem then boils down to that of matching the RSS levels obtained following an actual localization request with those in the training data set. A variety of approaches have been used for finding best match. As such, Radar [4] proposes a nearest neighbor search and employs Viterbi-like algorithm for further enhancement. On the other hand, [5] and Horus [6] treat the matching problem probabilistically. The Compass [7] also uses probabilistic approach and additionally accounts for antenna orientation. In terms of accuracy, the experimental tests of Radar and Compass conduced in a $312 \mathrm{~m}^{2}$ indoor area yielded average localization errors of 2.26 and $1.65 \mathrm{~m}$, respectively [2].

Fingerprinting is usually time and labor intensive, which eventually increases the cost. In addition, the measured RSS values depend on room occupancy, locations of furniture and positions of access points. Consequently, a new fingerprinting activity is required whenever something changes in the indoor area, which is impractical and costly. The methods proposed to alleviate these issues include building radio maps in a semifingerprinting fashion [8] and replacing fingerprinting by using ray tracing [9]. With the similar goal, machine learning [10] and Gaussian processes [11] have been explored to completely avoid fingerprinting and perform indoor localization in nonparametric fashion.

Most of the parametric RSS based methods rely on a standard path loss model, where path loss coefficient is a model parameter [12]. The unknown path loss coefficient is obtained via power law fit in [13] and $1 \mathrm{~m}$ accuracy in a 4-by-3 $\mathrm{m}$ room with 5 access points is reported. The performance results of some other parametric and semi-parametric RSS based localization techniques are reported in [14] and [15]. More recent algorithms jointly estimate unknown location and path loss coefficints. As such, the method in [16] assumes that the path loss coefficients are the same for all access points at a given location and estimates it jointly with an unknown location by formulating and solving a corresponding nonlinear least squares problem. In comparison, [17] considers different path loss coefficients associated with each access point and defines a geometric-based function which quantifies 
a compatibility between estimates of the location and path loss coefficients. The joint estimation is carried out by optimizing the compatibility function using the least squares method.

We propose in this paper an unsupervised, WiFi RSS based indoor localization method which relies on path loss model and jointly estimates an unknown location and path loss coefficients associated with different access points. We assume that the number of access points and their locations in the area are known. In comparison to geometric approach in [17], we treat path loss coefficients as random variables which take values from some finite, pre-defined alphabet. The algorithm carries over joint estimation by employing the Expectation Maximization (EM) procedure. The proposed algorithm is of low complexity and has been experimentally tested in an office space environment during business hours. The testing area has a rectangular shape of dimensions $32-\mathrm{by}-52 \mathrm{~m}$, with the total area of $1600 \mathrm{~m}^{2}$, and five access points provide WiFi signal coverage. The average localization accuracy achieved with the proposed method is below $4.5 \mathrm{~m}$.

A fair comparison between different indoor localization methods is somewhat difficult task because the accuracies reported in the literature are measured in indoor areas which have different layouts, dimensions, occupancies and the number of access points. A recently held competition tested a variety of methods under the same conditions and in an indoor area of $300 \mathrm{~m}^{2}$ with 10 access points [3]. The best performing infrastructure-free methods use WiFi fingerprinting and some of them enhance the estimation accuracy by employing the measurements obtained from the Inertial Measurement Unit (IMU). The achieved average localization errors are in the range between 1.6 and $2.81 \mathrm{~m}$. In comparison with that result, out method achieves around two times larger localization error in more than five times larger area and with half the number of access points.

\section{Proposed Algorithm}

\section{A. Setup and Model}

We assume that $N$ access points are placed throughout an indoor area of interest. The location of the $j$-th access point in a coordinate system associated with the indoor area is $\mathbf{x}_{j}=$ $\left(x^{(j)}, y^{(j)}, z^{(j)}\right)$, where $j=1, \ldots, N$. An access point $j$ is characterized with the reference received signal strength (RSS) level $z_{j}^{R}$, measured at the reference distance $d_{0}$. We assume that the locations of the access points $\mathbf{x}_{j}$ are known. As it will become clear later, we do not rely on accurate knowledge of the reference RSS levels $z_{j}^{R}$.

A level of the signal received from access points $j$ at some unknown location $\mathbf{x}$ is denoted with $z_{j}$, where $j=1, \ldots, N$. A path loss model describes the relation between RSS measurement $z_{j}$ and location $\mathbf{x}$ as [12]

$$
z_{j}=z_{j}^{R}-10 h_{j} \log _{10} \frac{\left\|\mathbf{x}-\mathbf{x}_{j}\right\|}{d_{0}}+v_{j},
$$

where $h_{j}$ is path loss coefficient and $v_{j}$ is measurement noise corresponding to the access point $j$. The path loss coefficient quantifies how quickly the radiated signal power decays as the distance from the access point increases. In free space, $h=2$. It usually has higher values in more complicated settings such as indoor areas.

The power of the received signal is well modeled as a lognormal probability distribution and thus the measurement noise $v_{j}$ is assumed to be Gaussian distributed with zero mean and variance $\sigma_{v}^{2}$. To keep expressions uncluttered, the variance of the measurement noise is assumed to be the same for all access points. The extension to a more general case is straightforward.

Given the RSS measurements $z_{j}, j=1, \ldots, N$, taken at some unknown location $\mathbf{x}$, our goal is to estimate the coordinates of this location using path loss model (1). In the following, we first consider the case when the path loss coefficients are known, and then study a more realistic case when they are unknown. We point out that one path loss coefficient is associated with each access point at each location.

\section{B. Known Path Losses}

With the assumption that the measurement noise $v_{j}$ is Gaussian, path loss model (1) yields that the RSS measurement $z_{j}$, conditioned on the path loss coefficient $h_{j}$ and location $\mathbf{x}$, is Gaussian distributed such that

$$
p\left(z_{j} \mid \mathbf{x}, h_{j}\right) \sim \mathcal{N}\left(z_{j}^{R}-10 h_{j} \log _{10} \frac{\left\|\mathbf{x}-\mathbf{x}_{j}\right\|}{d_{0}}, \sigma_{v}^{2}\right),
$$

where $p(a \mid b)$ is the probability density function of $a$ conditioned on $b$, while $\mathcal{N}(a, b)$ denotes Gaussian distribution of mean $a$ and variance $b$.

In addition, note that the RSS measurements $z_{j}$, corresponding to different access points $j$, are independent conditioned on location $\mathrm{x}$. Therefore, the joint log-likelihood of the RSS measurements is given by

$$
\begin{aligned}
& l\left(z_{1}, \ldots, z_{N} \mid \mathbf{x}, h_{1}, \ldots, h_{N}\right)= \\
& -\frac{1}{2 \sigma_{v}^{2}} \sum_{j=1}^{N}\left(z_{j}-z_{j}^{R}+10 h_{j} \log _{10} \frac{\left\|\mathbf{x}-\mathbf{x}_{j}\right\|}{d_{0}}\right)^{2} .
\end{aligned}
$$

Consequently, the maximum likelihood (ML) estimate of the unknown location $\mathrm{x}$ is obtained from the joint loglikelihood (3) as

$$
\hat{\mathbf{x}}_{\mathrm{ML}}=\underset{\mathbf{x}}{\operatorname{argmax}} l\left(z_{1}, \ldots, z_{N} \mid \mathbf{x}, h_{1}, \ldots, h_{N}\right)
$$

\section{Unknown Path Losses}

The path loss coefficients are unknown and location dependent because a single obstacle which prevents a direct line-ofsight (LOS) to an access point may change the corresponding path loss coefficient dramatically. Thus, to estimate an unknown location using the path loss model, the path loss coefficients also need to be inferred.

In general, a path loss coefficient is a continuos variable. However, for the reasons that will become clear later, we model a path loss coefficient as a discrete random variable which takes values from some finite, pre-defined, alphabet $\mathcal{H}$. The probability distribution of the path loss coefficient $h_{j}$ 


$$
\mathbf{E}_{p_{j}^{(k-1)}(h)}\left[l\left(z_{1}, \ldots, z_{N} \mid \mathbf{x}, h_{1}, \ldots, h_{N}\right)\right]=-\frac{1}{\sigma_{v}^{2}} \sum_{j=1}^{N} \sum_{h \in \mathcal{H}} p_{j}^{(k-1)}(h)\left(z_{j}-z_{j}^{R}+10 h \log _{10} \frac{\left\|\mathbf{x}-\mathbf{x}_{j}\right\|}{d_{0}}\right)^{2}
$$

corresponding to access point $j$, is denoted by $p_{j}(h)$, where $j=1, \ldots, N$ and $h \in \mathcal{H}$.

Without loss of generality, we implicitly assume that path loss coefficients corresponding to different access points take values from the same alphabet. The size of alphabet $\mathcal{H}$ should be large enough such that the RSS measurements accurately fit path loss model (1). On the other hand, the computational complexity scales with the size of $\mathcal{H}$.

Given this setup, the Expectation Maximization (EM) algorithm is used to iteratively estimate path loss coefficients and the unknown location [18]. Without any prior information, we initialize the EM algorithm with the uniform distribution on path loss coefficients

$$
p_{j}^{(0)}(h)=\frac{1}{|\mathcal{H}|}, h \in \mathcal{H}, j=1, \ldots, N,
$$

where the superscript ${ }^{()}$denotes the iteration index.

The $k$-th iteration of the EM algorithm starts with evaluating the expectation of the joint log-likelihood with respect to probability distributions of model parameters $h_{j}, j=$ $1, \ldots, N$, obtained in the previous iteration. Therefore, taking the expectation of (3) and treating $h_{j}$ 's as random variables with distributions $p_{j}^{(k-1)}(h), j=1, \ldots, N$, evaluated in the previous iteration $(k-1)$, the expected joint log-likelihood is given by (6).

The estimate of the unknown location at iteration $k$, is updated in the $\mathrm{M}$ step by maximizing the expected loglikelihood (6). Swapping the sign, the location estimate at iteration $k$ is evaluated as

$$
\hat{\mathbf{x}}^{(k)}=\underset{\mathbf{x}}{\operatorname{argmin}} C,
$$

where the objective function $C$ is given by

$$
C=\sum_{j=1}^{N} \sum_{h \in \mathcal{H}} p_{j}^{(k-1)}(h)\left(z_{j}-z_{j}^{R}+10 h \log _{10} \frac{\left\|\mathbf{x}-\mathbf{x}_{j}\right\|}{d_{0}}\right)^{2} .
$$

The optimization problem (8) is solved by using one of the gradient based optimization techniques for nonlinear programming [19]. Note that the objective function admits closed form expression for the gradient and Hessian. Also, the objective function is non-convex and a possible approach to partially overcome it would be to start a certain number of optimization procedures with different initial points and run them in parallel. Once all procedures converge, the solution which minimizes the objective function is chosen as the final estimate. Alternatively, one can perform a greedy search over the indoor area of interest. The greedy search is justified if the area of interest is not too large. A step size in the greedy search of about $1 \mathrm{~m}$ is a reasonable choice because the accuracy of the RSS based localization is most often above $1 \mathrm{~m}$.
The probability distribution $p_{j}(h), j=1, \ldots, N$, required for the E step of the next iteration, is updated using the location estimate $\mathbf{x}^{(k)}$. This distribution is effectively the probability distribution of the path loss coefficient $h_{j}$, conditioned on the location $\mathbf{x}$ and measured RSS level $z_{j}$, and is using the Bayes' rule given by

$$
p\left(h_{j} \mid \mathbf{x}, z_{j}\right) \propto p\left(z_{j} \mid \mathbf{x}, h_{j}\right) p\left(h_{j} \mid \mathbf{x}\right),
$$

where the likelihood $p\left(z_{j} \mid \mathbf{x}, h_{j}\right)$ is given by (2).

The conditional distribution $p\left(h_{j} \mid \mathbf{x}\right)$ essentially represents our current knowledge about the path loss $h_{j}$, which is summarized in $p_{j}^{(k-1)}(h)$. However, recalling the concept of extrinsic information, successfully applied in turbo coding and equalization [20], a uniform prior on path loss coefficient $h_{j}$ is used in place of $p\left(h_{j} \mid \mathbf{x}\right)$. We point out that $p_{j}^{(k-1)}(h)$ has already been used in the E step of the current iteration and using this distribution again would lead to overuse of the same information.

Therefore, the probability distribution of path loss coefficient $j$ is up to normalization constant given by

$$
\begin{aligned}
& p_{j}^{(k)}(h) \propto p\left(z_{j} \mid \mathbf{x}, h_{j}\right) \propto \\
& \propto \exp \left\{-\frac{1}{2 \sigma_{v}^{2}}\left(z_{j}-z_{j}^{R}+10 h \log _{10} \frac{\left\|\hat{\mathbf{x}}^{(k)}-\mathbf{x}_{j}\right\|}{d_{0}}\right)^{2}\right\},
\end{aligned}
$$

where $h \in \mathcal{H}$ and $j=1, \ldots, N$. The normalization yields the updates of probability distributions $p_{j}^{(k)}(h)$ of path loss coefficients corresponding to each of $N$ access points. To avoid underflow issue, this computation should be done in the logarithmic domain.

The EM routine is carried out a predefined number of iterations $I_{\max }$. Alternatively, we can formulate a stopping criterion for checking if the algorithm converged. For example, the convergence can be declared if the difference between the location estimates obtained in consecutive iterations falls below a certain threshold.

As a final remark, we point out that the described algorithm is not sensitive to accurate knowledge of the reference RSS levels $z_{j}^{R}, j=1, \ldots, N$. Essentially, lack of information on reference RSS values can be overcome by choosing a broader range of values for path loss coefficients in the alphabet $\mathcal{H}$. In fact, this approach is used in our experimental testing.

We conclude this section with the summary of the proposed indoor localization method in Algorithm 1.

\section{EXPERIMENTAL TESTING}

The proposed indoor localization method is experimentally tested in our office building. This section first describes the experimental setup and then presents performance results corresponding to two collected data sets. 


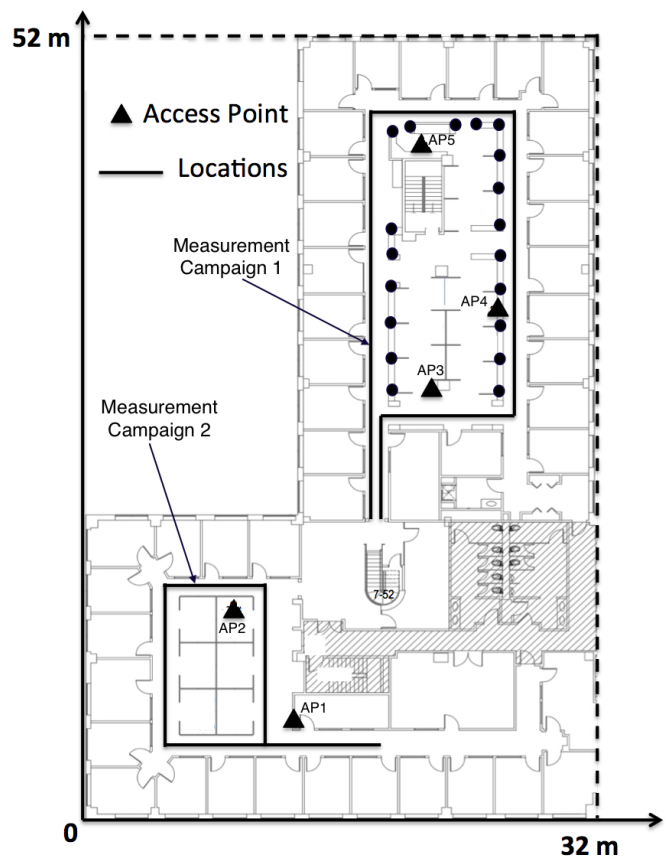

Fig. 1: Floor plan of the office space.

Algorithm 1: RSS based Indoor Localization Method

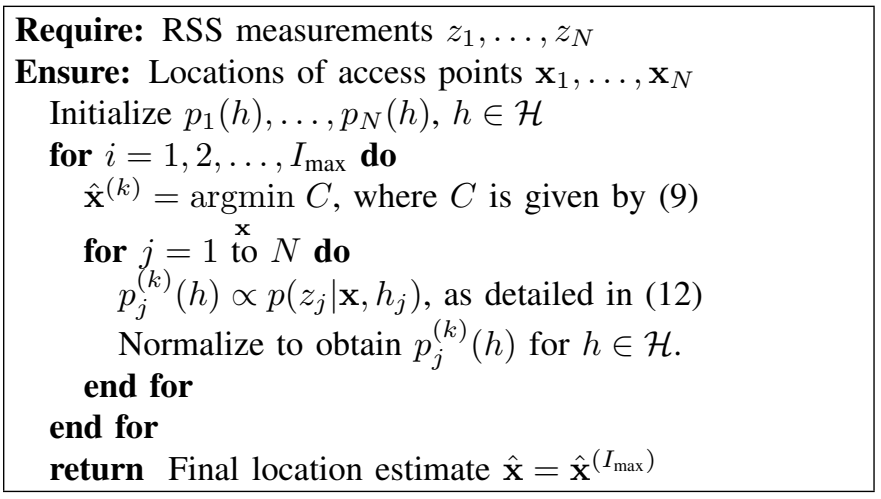

\section{A. Experimental Setup}

The accuracy of the proposed localization method is measured in the Mitsubishi Electric Research Labs' office space, whose floorplan is shown in Fig. 1. The considered indoor area consists of offices, cubicles, labs with different equipment, hallways and common areas. The cubicles in the upper left part of the office area are strengthen with a number of thick metal poles represented with black circles in Fig. 1. In addition, a number of storage metal boxes are placed in the hallways throughout the area (not shown in the floor plan).

The WiFi signal coverage in the considered area is provided from five access points whose positions are shown in Fig. 1. Note that the dimensions of the access points do not scale with the dimensions in the floor plan.

Two measurement campaigns were conducted on two different days and times in a week during the working hours. The

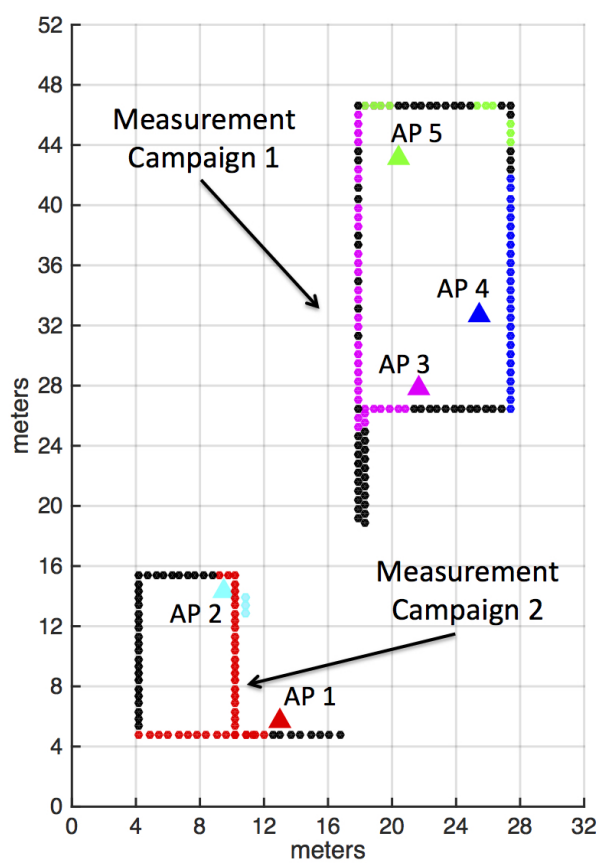

Fig. 2: Color-coded location indicating existence of LOS.

TABLE I: Heights of the access points with respect to the height of the RSS module.

\begin{tabular}{|l|r|r|r|r|}
\hline AP1 & AP2 & AP3 & AP4 & AP5 \\
\hline $1.49 \mathrm{~m}$ & $0.37 \mathrm{~m}$ & $1.58 \mathrm{~m}$ & $0.2 \mathrm{~m}$ & $0.39 \mathrm{~m}$ \\
\hline
\end{tabular}

campaigns took place in two different areas in the considered office space. A traffic common for an office space during a working day was observed. In addition, a construction in the area close to access point AP3 was happening during measurement campaign 1 (whose locations came close to the construction area in the beginning and end). The proposed algorithm is tested on two data sets separately because the measurements were taken at different days and times.

In each measurement campaign, a person is walking, taking RSS measurements and recording his true locations. The traversed paths enclose loops in clock-wise direction and are shown in Fig. 1. The coordinate system, positions of the measurement locations and positions of five access points, along with their labeling, are shown in Fig. 2. The true measurement locations and positions of the access points are measured with respect to the coordinate system associated with the area using a regular tape. The RSS levels are measured with a particular WiFi module which operates at $2.4 \mathrm{GHz}$, extracts beacons associated with access points from the received signal and outputs the RSS level for each access point, along with its identifiers (MAC address and SSID). The measured RSS level is expressed in $\mathrm{dBm}$ with the resolution of $1 \mathrm{dBm}$. The heights of the access points with respect to the RSS measuring module are given in Table I.

To give more details about the propagation environment, the measurement locations in Fig. 2 are color-coded such that 
the existence of line-of-sight (LOS) between a location and an access point is indicated by shading the two using the same color. The locations that do not have LOS with any of the access points are shaded in black. Note that some locations close to access point AP3 lose LOS to AP3 due to think metal poles shown in Fig.1. We also note that only three locations have LOS with access point AP2 because the AP2 is placed in a cubicle at lower height. These three locations also have LOS with access point AP1 and they are represented with two colors in Fig. 2.

The proposed localization algorithm is supplied with floor plan which defines a set of constraints in optimization problem (8). The search space for our experimental verification is a $32-$ by $-52 \mathrm{~m}$ rectangle between the axes and dashed lines shown in Fig. 1. Effectively, we assume no prior knowledge of where the walls are. In addition, the search area is larger than that implied by the floor plan. By doing this, we show that the algorithm is capable of estimating unknown locations in a larger indoor area with relatively small number of access points.

As a final remark, we note that the algorithm does not exploit the fact that the RSS measurements are taken at consecutive locations. That is, each location is treated independently and location estimate at a particular localization request is not used for localization in the following requests. Fusion of this possibility into the proposed method is left for future consideration.

\section{B. Experimental Results}

The RSS measurements taken at two measurement campaigns are plotted in Figures 3 and 4. As expected, the measured RSS levels closely follow the distance pattern between measurement locations and corresponding access points so that the signals transmitted from distant access points are weak and get close to the measured noise floor of $-94 \mathrm{dBm}$.

An impact of random shadowing, cased by people passing by, can be observed from the measured RSS levels at the beginning and end of measurement campaign 1 . As can be seen from Fig. 2, 10 measurement locations at the beginning and end of the campaign are aligned along parallel lines which are spaced by $\sim 0.5 \mathrm{~m}$. However, the RSS levels measured along these lines differ as much as $\sim 10 \mathrm{dBm}$, as can be observed from Fig. 3.

The proposed algorithm estimates indoor location based on the measured RSS levels taken at that location. The path loss coefficients take values form finite alphabet $\mathcal{H}=$ $\{2,2.5,3,3.5,4\}$. The EM routine converges after 2 iterations. The estimation performance is quantified with localization error, defined as the distance between the estimated and true location. The plots of the localization errors versus location index for two measurement campaigns are shown in Fig. 5. The empirical cumulative distribution functions (cdf's) corresponding to two measurement campaigns are shown in Fig. 6. The statistics of the localization errors from two campaigns, in particular 25\%,50\%, 75\% and $90 \%$ percentiles, as well as the average, are summarized in Table II. To conclude, the average

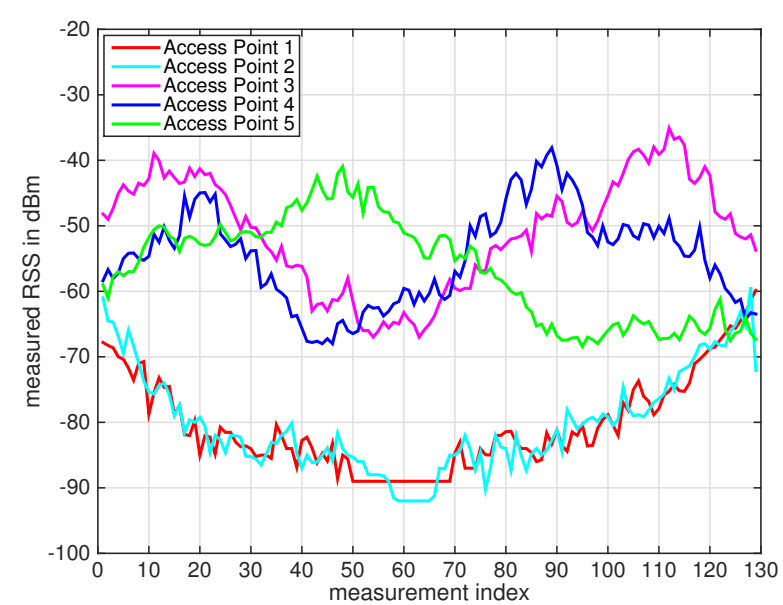

Fig. 3: Measured RSS levels in measurement campaign 1.

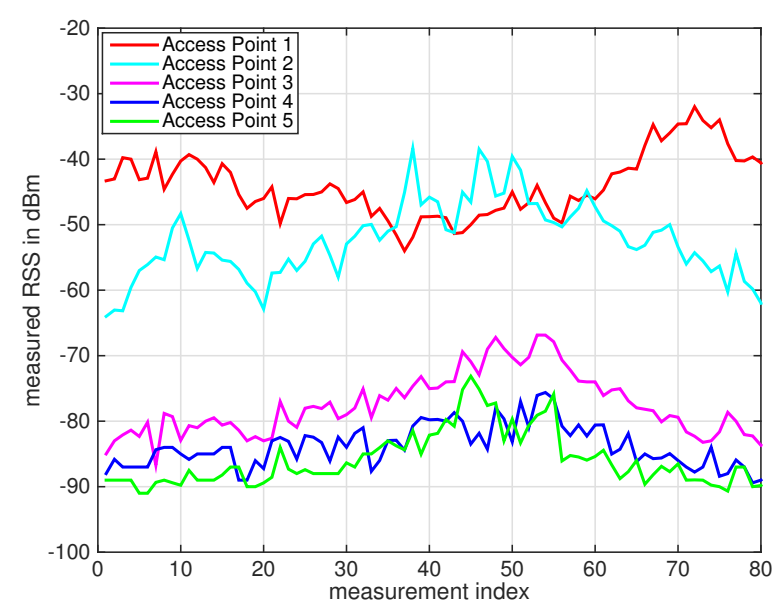

Fig. 4: Measured RSS levels in measurement campaign 2.

localization error is below 4.5 meters in both measurement campaigns.

\section{CONCLUSION}

We propose in this paper an indoor localization algorithm which estimates unknown location based on the measured strengths of the signals received from the WiFi access points. The algorithm is unsupervised in nature. In addition, it solely relies on existing WiFi chipsets and does not require any change on the hardware side. The algorithm is developed by modeling the measured RSS level using the path loss model, where path loss coefficient corresponding to each access point and at a given location is modeled as a discrete

TABLE II: Summary of the localization error statistics.

\begin{tabular}{|l|r|r|r|r|r|}
\hline percentiles & $25 \%$ & $50 \%$ & $75 \%$ & $90 \%$ & mean \\
\hline Campaign 1 & $3.44 \mathrm{~m}$ & $4.04 \mathrm{~m}$ & $5.32 \mathrm{~m}$ & $6.33 \mathrm{~m}$ & $\mathbf{4 . 4 6} \mathbf{~ m}$ \\
\hline Campaign 2 & $2.65 \mathrm{~m}$ & $4.38 \mathrm{~m}$ & $6.06 \mathrm{~m}$ & $6.63 \mathrm{~m}$ & $\mathbf{4 . 3 6} \mathbf{~ m}$ \\
\hline
\end{tabular}



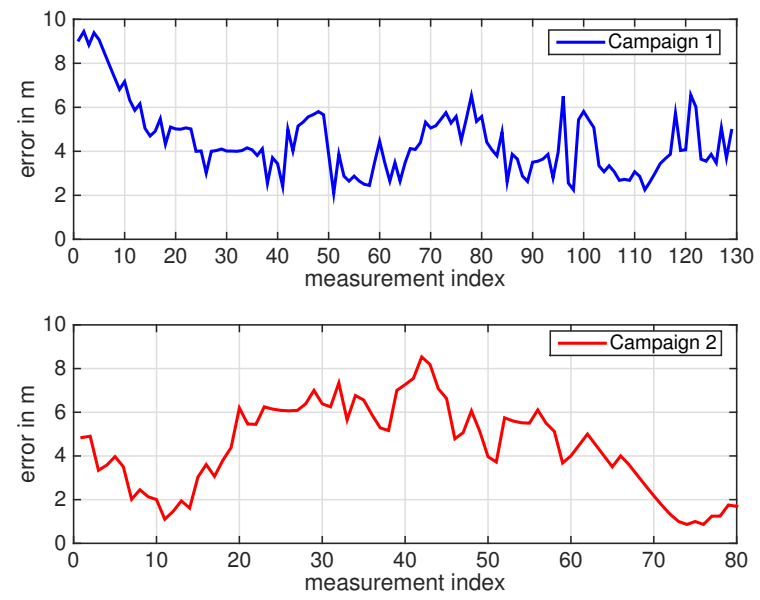

Fig. 5: Localization error versus location index for campaign 1 (top figure) and campaign 2 (bottom figure).

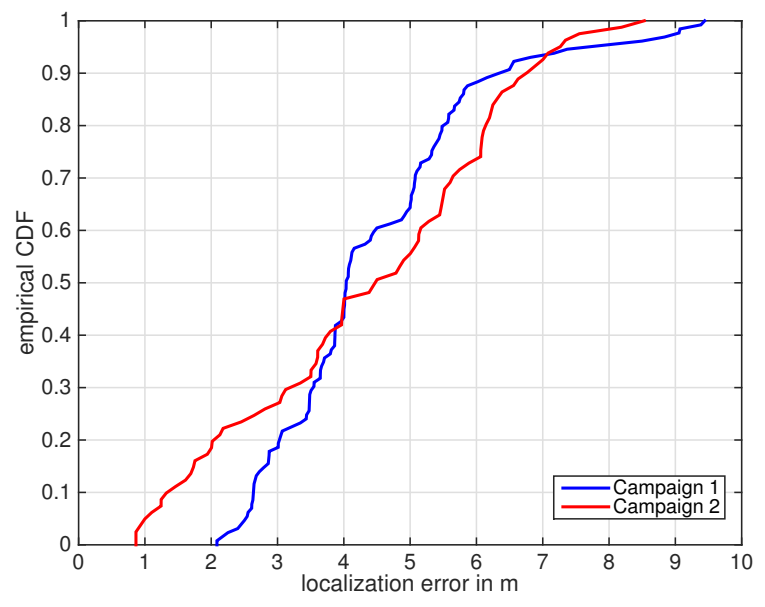

Fig. 6: Empirical CDF of localization error for two measurement campaigns.

random variable which takes values from a finite alphabet. Both an unknown location and path loss coefficients are jointly estimated using the Expectation Maximization (EM) procedure. The proposed method was experimentally tested in a $32-$ by $-52 \mathrm{~m}$ (i.e., $1600 \mathrm{~m}^{2}$ ) office space area where five access points provide WiFi signal coverage. Two experimental tests were conduced at two different times, days and places in the office area, and the attained average localization error was below $4.5 \mathrm{~m}$ in both tests.

\section{ACKNOWLEDGMENT}

The authors would like to thank Takuya Fujihashi and Kapil Borle for their help in writing code and taking measurements.

\section{REFERENCES}

[1] H. Liu, H. Darabi, P. Banerjee, and J. Liu, "Survey of wireless indoor positioning techniques and systems," Systems, Man, and Cybernetics,
Part C: Applications and Reviews, IEEE Transactions on, vol. 37, no. 6, pp. 1067-1080, November 2007.

[2] Y. Gu, A. Lo, and I. Niemegeers, "A survey of indoor positioning systems for wireless personal networks," Communications Surveys Tutorials, IEEE, vol. 11, no. 1, pp. 13-32, First quarter 2009.

[3] D. Lymberopoulos, J. Liu, X. Yang, R. R. Choudhury, V. Handziski, and S. Sen, "A realistic evaluation and comparison of indoor location technologies: Experiences and lesson learnt," 14th International Conference on Information Processing in Sensor Networks (IPSN '15), Seattle, WA, April 2015.

[4] P. Bahl and V. N. Padmanabhan, "RADAR: An in-building RF-based user location and tracking system," INFOCOM 2000. Nineteenth Annual Joint Conference of the IEEE Computer and Communications Societies. Proceedings. IEEE, vol. 2, pp. 775-784, 2000.

[5] T. Roos, P. Myllymäki, H. Tirri, P. Misikangas, and J. Sievänen, "A Probabilistic Approach to WLAN User Location Estimation," International Journal of Wireless Information Networks, vol. 9, pp. 155-164, 2002.

[6] M. Youssef and A. Agrawala, "The Horus WLAN location determination system," MobiSys '05: Proceedings of the 3rd international conference on Mobile systems, applications, and services, pp. 205-218, 2005.

[7] T. King, S. Kopf, T. Haenselmann, C. Lubberger, and W. Effelsberg, "COMPASS: A probabilistic indoor positioning system based on 802.11 and digital compasses," Proc. First ACM Intl Workshop on Wireless Network Testbeds, Experimental evaluation and Characterization (WiNTECH), September 2006.

[8] D. Zou, W. Meng, S. Han, Z. Gong, and B. Yu, "User aided self-growing approach on radio map construction for WLAN based localization," Proceedings of the 26th International Technical Meeting of The Satellite Division of the Institute of Navigation (ION GNSS+ 2013), pp. 991-997, September 2013.

[9] M. Laaraiedh, B. Uguen, J. Stephan, Y. Corre, Y. Lostanlen, M. Raspopoulos, and S. Stavrou, "Ray tracing-based radio propagation modeling for indoor localization purposes," Computer Aided Modeling and Design of Communication Links and Networks (CAMAD), 2012 IEEE 17th International Workshop on, pp. 276-280, September 2012.

[10] R. Faragher and R. Harle, "SmartSLAM - an efficient smartphone indoor positioning system exploiting machine learning and opportunistic sensing," Proceedings of the 26th International Technical Meeting of The Satellite Division of the Institute of Navigation (ION GNSS+ 2013), pp. 1006 - 1019, September 2013.

[11] B. Ferris, D. Fox, and N. Lawrence, "WiFi-SLAM using Gaussian process latent variable models," Proceedings of the 20th International Joint Conference on Artifical Intelligence, pp. 2480-2485, 2007.

[12] A. Goldsmith, Wireless Communications. Cambridge University Press, 2005.

[13] N. Dieng, C. Chaudet, M. Charbit, L. Toutain, and T. Ben Meriem, "Experiments on the RSSI as a range estimator for indoor localization," New Technologies, Mobility and Security (NTMS), 2012 5th International Conference on, pp. 1-5, May 2012.

[14] G. Zanca, F. Zorzi, A. Zanella, and M. Zorzi, "Experimental comparison of RSSI-based localization algorithms for indoor wireless sensor networks," Proceedings of the workshop on Real-world wireless sensor networks, pp. 1-5, 2008.

[15] P. Pivato, L. Palopoli, and D. Petri, "Accuracy of RSS-based centroid localization algorithms in an indoor environment," Instrumentation and Measurement, IEEE Transactions on, vol. 60, no. 10, pp. 3451-3460, October 2011.

[16] X. Li, "RSS-based location estimation with unknown pathloss model," Wireless Communications, IEEE Transactions on, vol. 5, no. 12, pp. 3626-3633, December 2006.

[17] S. Mazuelas, A. Bahillo, R. Lorenzo, P. Fernandez, F. Lago, E. Garcia, J. Blas, and E. Abril, "Robust indoor positioning provided by real-time RSSI values in unmodified WLAN networks," Selected Topics in Signal Processing, IEEE Journal of, vol. 3, no. 5, pp. 821-831, October 2009.

[18] C. Bishop, Pattern Recognition and Machine Learning, 1st ed. SpringerVerlag New York, 2006.

[19] D. Bertsekas, Nonlinear Porgramming, 2nd ed. Athena Scientific, September 1999.

[20] M. Tüchler, R. Koetter, and A. C. Singer, "Turbo equalization: Principles and new results," Communications, IEEE Transactions on, vol. 50, no. 5, pp. 754-767, May 2002. 